\title{
Aktuelle News vom ASCO-GI 2018
}

Der diesjährige ASCO-GI bot einige äußerst interessante und zum Teil auch «practice-changing» Studienergebnisse. Im Folgenden ein kleiner Querschnitt durch die Highlights bei den gastrointestinalen Tumoren.

\section{Magen- und Ösophaguskarzinom}

Nachdem Ramucirumab bei Patienten mit fortgeschrittenem Magenkarzinom in der Zweitlinie sowohl als Monotherapie als auch in Kombination mit Paclitaxel zu einer signifikanten Verbesserung des Gesamtüberlebens (OS) führte, erfolgte nun in der RAINFALL-Studie [1] der Einsatz des monoklonalen VEGFR-2-Antikörpers in der Erstlinientherapie. In dieser Phase-III-Studie erhielten 645 Patienten Ramucirumab in Kombination mit Chemotherapie oder Chemotherapie alleine. Der primäre Endpunkt progessionsfreies Überleben (PFS) war im Ramucirumab-Arm signifikant später erreicht als im Vergleichsarm, wobei der Unterschied von 0,3 Monaten im medianen PFS insgesamt gering ausfiel (5,7 vs. 5,4 Monate; $p=0,011)$. Beim OS und Ansprechen ergab sich nur ein numerischer Vorteil für Ramucirumab. Behandlungsdauer und Postprogressionstherapie unterschieden sich nicht zwischen beiden Armen. Damit ist ein Nutzen der Antiangiogenese in der Erstlinientherapie des fortgeschrittenen Adenokarzinoms des Magens oder des gastroösophagealen Übergangs nicht belegt.

Interessant war auch die Analyse der Kohorte 1 aus der KEYNOTE059-Studie [2] mit dem PD-1-Inhibitor Pembrolizumab als Monotherapie bei Patienten mit mindestens 2 Vortherapien eines metastasierten Magenkarzinoms. Das Ansprechen lag in der Gesamtkohorte ( $\mathrm{n}=$ 259 ) bei $12 \%$ und in der Gruppe mit PD-L1-positiven Tumoren ( $\mathrm{n}=$ 148) mit 16\% etwas höher. Die Ansprechdauer war sowohl in der Gesamtkohorte wie auch in der PD-L1-positiven Gruppe 14 Monate. Bei den 7 Patienten mit ausgeprägter Mikrosatelliteninstabilität zeigte sich eine Ansprechrate von 57\%, wobei die mediane Ansprechdauer noch nicht erreicht war.

\section{Hepatozelluläres Karzinom}

Nach positiven Ergebnissen aus einer Phase-II-Studie [3] wurde Cabozantinib, ein dualer MET/VEGFR2-Inhibitor, aktuell in der Phase-III-Studie CELESTIAL [4] bei 773 Patienten mit fortgeschrittenem Sorafenib-refraktären hepatozellulären Karzinom (HCC) eingesetzt. Cabozantinib senkte das Sterberisiko signifikant um $24 \%$ gegenüber Best Supportive Care (medianes OS: 10,2 vs. 8 Monate; $\mathrm{p}=$ 0049). Das mediane PFS lag bei 5,2 Monaten versus 1,9 Monate (HR = 0,44; $\mathrm{p}<0,0001)$. Die häufigsten Grad-3/4-Toxizitäten unter Cabozantinib waren Hand-Fuß-Syndrom (17\%), Hypertonie (16\%), Anstieg der Leberenzyme (12\%), Fatigue (10\%) und Diarrhö (10\%). Die Therapieabbruchrate wegen Nebenwirkungen lag bei $16 \%$. Damit stellt
Cabozantinib eine neue Therapieoption in der Zweitlinie des fortgeschrittenen HCC dar.

Mit der KEYNOTE-224-Studie [5] konnte für Pembrolizumab als Monotherapie beim fortgeschrittenen Sorafenib-refraktären HCC in der Zweit- und Drittlinientherapie eine beachtliche Antitumor-Aktivität gezeigt werden. 105 Patienten wurden in die einarmige Phase-IIStudie eingeschlossen. Die Gesamtansprechrate lag bei 16,3\%, darunter eine komplette Remission. 15,4\% der Patienten erreichten eine partielle Remission und 45,2\% eine Stabilisierung. Die Krankheitskontrollrate betrug 61,5\%. Das mediane PFS lag bei 4,8 Monaten und das mediane OS war noch nicht erreicht. Nach 6 Monaten ergab sich eine PFS- und OS-Rate von 43,1 bzw. 77,9\%. Es gab keine neuen Sicherheitssignale, insbesondere auch keine viralen Flares. 23 Patienten standen zum Zeitpunkt der Analyse noch unter der Behandlung. Pembrolizumab wird derzeit in einer Phase-III-Studie versus Placebo bei vorbehandelten HCC-Patienten mit OS als primären Endpunkt untersucht (KEYNOTE-240).

\section{Kolorektalkarzinom}

Beim fortgeschrittenen Kolonkarzinom gilt der Multikinase-Inhibitor Regorafenib als effektiver Standard in der Drittlinientherapie, der allerdings mit einer nicht unerheblichen Toxizität einhergeht. In der prospektiven Phase-II-Studie ReDOS [6] wurde daher Regorafenib in der Standarddosis $160 \mathrm{mg}$ täglich (Tag 1-21) gegenüber einem wöchentlichen Dosiseskalationskonzept für den ersten Behandlungszyklus mit täglich $80 \mathrm{mg}$ in Woche $1,120 \mathrm{mg}$ in Woche 2 und $160 \mathrm{mg}$ in Woche 3 verglichen. Insgesamt wurden 123 Patienten in die Studie eingebracht. Im Eskalationsarm zeigte sich nicht nur eine deutlich bessere Verträglichkeit, sondern auch eine zumindest vergleichbare und tendenziell sogar bessere Wirksamkeit, bei insgesamt erreichter höherer Dosisintensität. Im OS ergab sich ein tendenzieller, aber nicht signifikanter Vorteil (9 vs. 5,9 Monate; HR = 0,65; p = 0,09). Der Unterschied im PFS betrug 2,5 Monate im Eskalationsarm versus 2 Monate im Standardarm. In der täglichen Praxis scheint demnach ein Eskalationskonzept sinnvoll und könnte sogar einen Überlebensvorteil bedeuten.

Dr. med. Gerhard Emrich

\section{Literatur}

1 Fuchs CS, et al: ASCO-GI 2018;abstr 5.

2 Muro K, et al: ASCO-GI 2018;abstr 723.

3 Kelley RK, et al: Cabozantinib in hepatocellular carcinoma: results of a phase 2 placebo-controlled randomized discontinuation study. Ann Oncol 2017;28:528-534.

4 Abou-Alfa GK, et al: ASCO-GI 2018;abstr 207.

5 Zhu AX, et al: ASCO-GI 2018;abstr 209.

6 Bekaii-Saab TS, et al: ASCO-GI 2018;abstr 611. 